\title{
Polarization sensitive silicon photodiodes using nanostructured metallic grids
}

\author{
M. Guillaumée, ${ }^{1, a)}$ L. A. Dunbar, ${ }^{1}$ Ch. Santschi, ${ }^{2}$ E. Grenet, ${ }^{1}$ R. Eckert, ${ }^{1}$ O. J. F. Martin, ${ }^{2}$ \\ and R. P. Stanley ${ }^{1}$ \\ ${ }^{1}$ Swiss Centre for Electronics and Microtechnology, (CSEM SA), Jaquet-Droz, 1, CH-2002 Neuchâtel, \\ Switzerland \\ ${ }^{2}$ Nanophotonics and Metrology Laboratory, Ecole Polytechnique Fédérale de Lausanne (EPFL), \\ CH-1015 Lausanne, Switzerland
}

(Received 21 January 2009; accepted 23 April 2009; published online 12 May 2009)

\begin{abstract}
In this paper, we present the design, fabrication, and characterization of wire grid polarizers. These polarizers show high extinction ratios and high transmission with structure dimensions that are compatible with current complementary metal-oxide-semiconductor (CMOS) technology. To design these wire grids, we first analyze the transmission properties of single apertures. From the understanding of a single aperture, we apply a modal expansion method to model wire grids. The most promising grids are fabricated on both a glass substrate and CMOS photodiode. An extinction ratio higher than 200 is measured. (c) 2009 American Institute of Physics.
\end{abstract}

[DOI: $10.1063 / 1.3133862$ ]

Complementary metal-oxide-semiconductor (CMOS) image sensors are found in many devices, from mobile phone cameras to high performance industrial sensors. They are sensitive across the visible, and color information can be extracted through filters on individual pixels. Achieving this for polarization would provide information such as shape and object properties. ${ }^{1}$ For example, polarization imaging can improve biomedical imaging contrast due to the birefringence of biological tissue. ${ }^{2}$ Information on the polarization state is usually obtained by mechanically rotating a polarizer in front of a camera. Movable components can be avoided on integrating micropolarizers on each individual pixel, allowing to acquire polarization information at the pixel level. ${ }^{3}$ Similar to the Bayer configuration, a smart arrangement of pixels allows the reconstruction of the polarization state. ${ }^{4}$

Several types of micropolarizers have been investigated. ${ }^{3-5}$ Metallic wire grid polarizers (WGPs) (Ref. 6) would seem the obvious candidate, their advantages being high acceptance angle, broadband extinction ratio (ER), compactness, and potential CMOS integration. WGPs are used from radio-waves ${ }^{7}$ to the infrared. ${ }^{8}$ Their extension to the visible is challenging; to obtain high transmission and ER in a WGP, the pitch $p$ (Fig. 1) has to be much smaller than the incident wavelength $\lambda(p<\lambda / 10)$, i.e., $p<100 \mathrm{~nm} .{ }^{9} \mathrm{Im}$ proved fabrication techniques, such as nanoimprint lithography, enable the fabrication of wires as small as $50 \mathrm{~nm} .{ }^{10}$ It would be commercially interesting to fabricate these grids directly in the CMOS process, but this limits the minimum pitch. Tamada et al. ${ }^{11}$ showed that efficient polarization properties can however be obtained in the resonant regime (i.e., $p \approx \lambda$ ) by controlling both aperture width $w$ and film thickness $h$.

This letter presents efficient WGP for visible imaging working in the resonant regime. Polarization selectivity is first considered on a single slit. The transmission of WGP is calculated from a modal expansion method. Efficient WGPs

${ }^{a)}$ Electronic mail: mickael.guillaumee@csem.ch. are then fabricated and characterized on both glass and pixels of a CMOS sensor.

Consider first a 1D subwavelength aperture in a metallic film. Such an aperture always supports a propagating mode for $p$-polarized light (the polarization state is defined in Fig. 1). Due to reflection at the entrance and exit of the aperture, Fabry-Pérot (FP)-like behavior occurs for sufficiently thick films, and high transmission can be obtained at resonant wavelengths. ${ }^{12}$ On the other hand, $s$-polarized light supports only evanescent modes for small aperture widths. The amplitude of evanescent modes inside the aperture decays exponentially with distance from the entrance. Increasing $h$ consequently strongly reduces $s$-transmission. ${ }^{11}$ For perfect metals, $s$-polarized modes are nonpropagating for $\lambda>\lambda_{c}$, where $\lambda_{c}=w / 2$ is the cutoff wavelength. $\lambda_{c}$ cannot be sharply defined for real metals due to their finite conductivity. ${ }^{13}$

The influence of the aperture width on the ER is experimentally investigated for gold films on a glass substrate. The gold films are deposited by sputtering. Apertures are then milled with a focused ion beam (FIB). Samples are illuminated with a halogen lamp. The incoming beam has a low numerical aperture $(\mathrm{NA}<0.1)$. Its polarization state is controlled using a Glan laser prism $\left(\mathrm{ER}=10^{5}\right)$. The transmitted light is collected through a microscope objective $(\mathrm{NA}=0.6)$. The collected signal is analyzed using a monochromator and a cooled charge-couple-device camera.

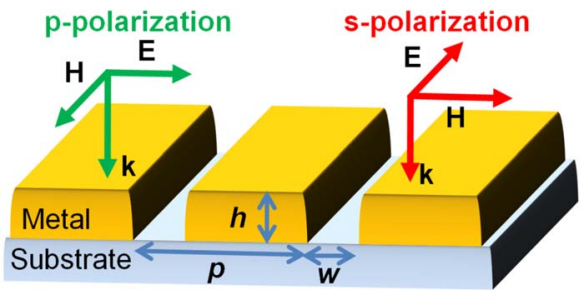

FIG. 1. (Color online) A schematic of a WGP on a glass substrate and definitions used in this letter for the polarization state and the grid dimensions. 


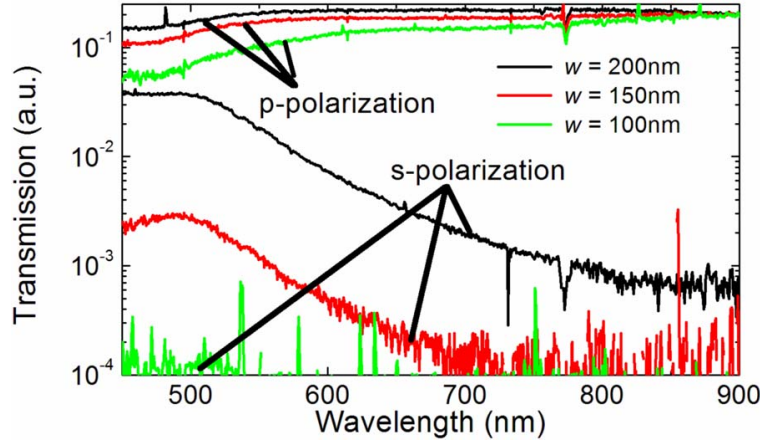

FIG. 2. (Color online) Transmission spectra of $10 \mu \mathrm{m}$ long single apertures etched in a $310 \mathrm{~nm}$ gold film for widths varying between 100 and $200 \mathrm{~nm}$.

Transmission measurements of single apertures in a 310 $\mathrm{nm}$ thick gold film are shown in Fig. 2 for widths varying from 100 to $200 \mathrm{~nm}$. In all cases, the highest transmission is observed for $p$-polarized light. The characteristic FP peak is indistinct due to the low reflection inside the aperture. Below $500 \mathrm{~nm}$, the transmission decreases for both polarizations due to the nonmetallic properties of gold at short wavelengths. ${ }^{14}$ For the $s$-polarization, the transmission decreases rapidly with increasing $\lambda$ or decreasing $w$, characteristic of transmission below cutoff. The $s$-polarized transmission is comparable to the instrumental noise for $100 \mathrm{~nm}$ slit width. In contrast, $p$-polarized transmission remains high at long $\lambda$. In all cases, the lowest ER occurs at short wavelengths and varies strongly with slit width, i.e., the ER ranges from two to three to at least 500 for widths of 200 and $100 \mathrm{~nm}$, respectively.

In the following sections, $w=150 \mathrm{~nm}$ is chosen as a compromise between structure size and high ER. The $0.13 \mu \mathrm{m}$ CMOS technology currently used for image sensor fabrication provides a minimum metal spacing of $200 \mathrm{~nm}$. Nevertheless, as CMOS technologies keep scaling and smaller feature sizes become available, it will be possible to fabricate the suggested structures in the near future; the minimum spacing in $0.09 \mu \mathrm{m} \mathrm{CMOS} \mathrm{technology} \mathrm{is} \mathrm{of} 140 \mathrm{~nm} .^{15}$ The film thickness has been chosen to be close to standard CMOS metal thickness.

Optical properties of infinite wire grids can be simulated using modal expansions with surface impedance boundary conditions at metal-dielectric interfaces. ${ }^{16}$ The dielectric con- stant of gold is taken from SOPRA database, ${ }^{17}$ whereas the refractive index of the glass substrate is considered to be constant (1.525). Calculated zero order transmission spectra for various wire grids with $w=150 \mathrm{~nm}, h=285 \mathrm{~nm}$, and periods ranging from 350 to $600 \mathrm{~nm}$ are reported in Fig. 3(a). The minima observed for $p$-polarized light is related to surface plasmon polariton excitation. ${ }^{18}$ The waveguide mode supported by the slits leading to FP resonances is the main transmission channel. There is a phase change upon reflection experienced by the waveguide mode at the entrance or exit of the aperture. ${ }^{18,19}$ This has a strong dispersion close to Wood-Rayleigh anomalies ${ }^{20}$ occurring at $\lambda=p n_{d} / m$, where $n_{d}$ is the refractive index of air or glass and $m$ is an integer. This causes the FP transmission peaks to redshift on increasing the pitch of the grating, as observed in Fig. 3(a), where the high FP peak $(T=95 \%)$ for $p=350 \mathrm{~nm}$ at long wavelength is shifted toward the infrared with increasing $p$. Conventional WGPs do not show these complex behaviors since their periods and thicknesses are small compared to the wavelength, and thus resonant phenomena are not present.

For the $s$-polarization case, the transmission spectrum of a wire grid is very similar to that of a single aperture [Fig. 3(b)]. Varying the period mainly modifies the magnitude of transmission due to a change in aperture number per area. Transmission for $p$-polarization is at least two orders of magnitude higher than $s$-polarization in the $600-900 \mathrm{~nm}$ range but only two to three times higher at the $p$-transmission minima, i.e., at $\lambda \approx 550 \mathrm{~nm}$ and $\lambda \approx 800 \mathrm{~nm}$ [Fig. 3(d)].

Transmission measurements of wire grids, fabricated and measured the same way as the single apertures, are shown in Fig. 3(c). Simulation and experiment are in good agreement [see Figs. 3(a) and 3(c)]. Finite difference time domain method was used but did not improve the agreement (not shown due to space restrictions). The major discrepancy [better observed on a log scale, see Fig. 3(b)] is the dips in transmission for $p$-polarized light at $\lambda \approx 550 \mathrm{~nm}$ and $\lambda \approx 800 \mathrm{~nm}$, which are more pronounced in the calculations. The differences arise because the theoretical treatment does not take into account the finite size of the wire grid [ten slits of $10 \mu \mathrm{m}$ length, see inset of Fig. 3(c)], ${ }^{14}$ the nonzero NA of illumination, surface roughness, nor imperfection in the structure profile. Interestingly, the experimental ER is higher than the theoretically predicted one at these dips [Fig. 3(d)].
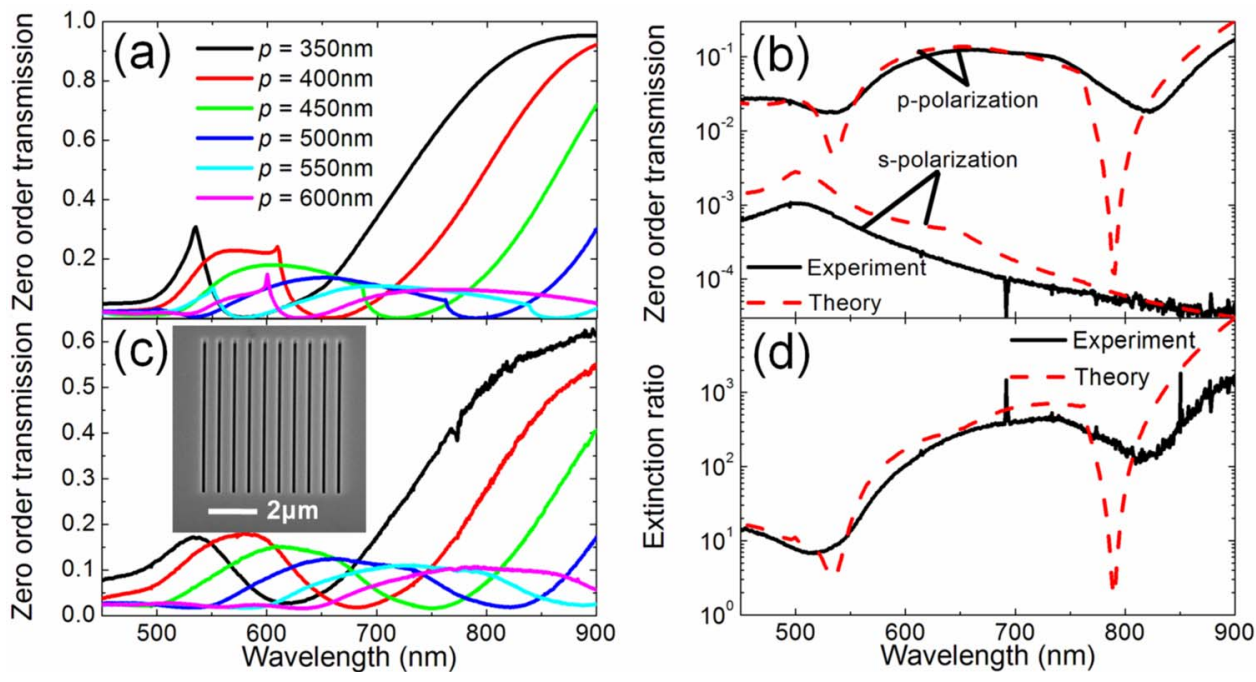

FIG. 3. (Color online) (a) Calculations of $p$-polarized zero order transmission of wire grids $(h=285 \mathrm{~nm}$ and $w$ $=150 \mathrm{~nm}$ ) for period varying between 350 and $600 \mathrm{~nm} \mathrm{(c)} \mathrm{and} \mathrm{corresponding}$ measurements. The inset in panel (c) is a SEM image of the wire grid with $p$ $=600 \mathrm{~nm}$. (b) $p$ - and $s$-polarized zero order transmission spectra (d) and the corresponding ER for $p=500 \mathrm{~nm}, h$ $=285 \mathrm{~nm}$, and $w=150 \mathrm{~nm}$. Black solid curves: experiment; red dashed curves: calculation. 


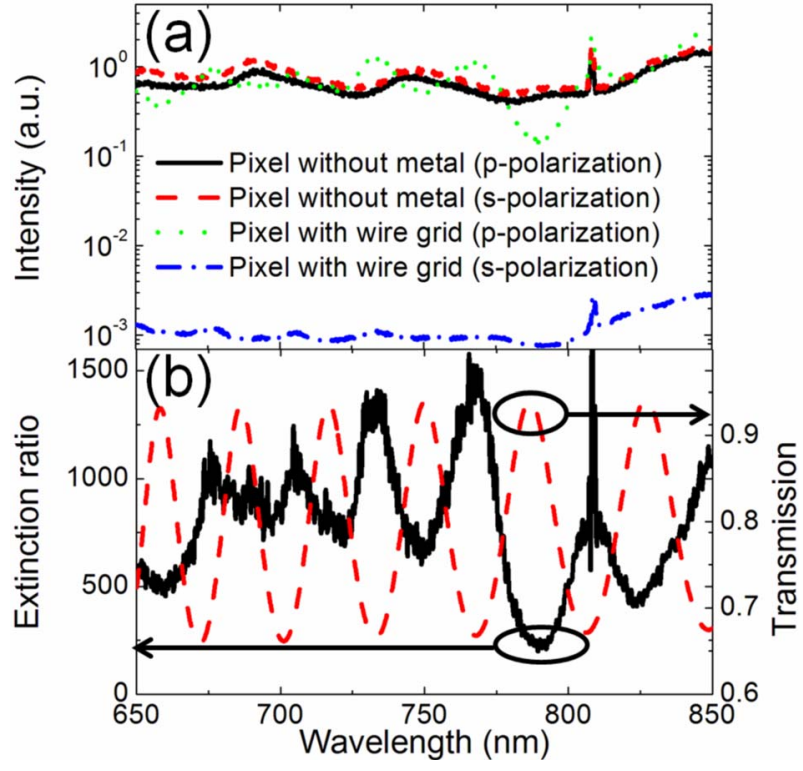

FIG. 4. (Color online) (a) Intensity measured for $p$ - and $s$-polarization on a pixel of a CMOS sensor without metal (black solid line and red dashed line, respectively) and with a wire grid with $p=500 \mathrm{~nm}, h=340 \mathrm{~nm}$, and $w=150 \mathrm{~nm}$ (green dotted line and blue dashed dotted line, respectively). (b) Corresponding ER (black solid line) and calculated transmission of a $5.375 \mu \mathrm{m}$ thick $\mathrm{SiO}_{x}$ layer on a silicon wafer.

A wire grid with $150 \mathrm{~nm}$ wide slits and a periodicity of $500 \mathrm{~nm}$ gives both an excellent ER and a good transmission in the visible-near infrared spectrum while having dimensions suitable for manufacturing. This design was integrated onto a pixel of a CMOS image sensor. ${ }^{21}$ The sensor consists of an array of $50 \times 50 \mu \mathrm{m}^{2}$ pixels, each pixel having an active area of $14 \times 14 \mu \mathrm{m}^{2}$. A $340 \mathrm{~nm}$ thick gold film was deposited by sputtering and apertures were milled using FIB technique. In the CMOS process used, a $5.5 \mu \mathrm{m}$ thick silicon oxide layer is present above the detector. Thus, refractive index below the grid is the same as for the glass substrate, and the transmission properties are expected to be similar. Measurements are performed with a supercontinuum light source coupled to a monochromator. Quasimonochromatic $(\Delta \lambda<2 \mathrm{~nm})$ light is focused on an individual pixel. The incident wavelength is scanned, while the signal of the pixel of interest is recorded. Two Glan laser prisms and a quarterwave plate are placed between the sample and the monochromator to control the polarization state.

Results are shown in Fig. 4. FP fringes are observed for pixels without metal due to the $\mathrm{SiO}_{x}$ layer above the active region. ${ }^{22}$ The WGP amplifies the oscillatory behavior. In addition, the oscillations undergo a $\pi$ phase shift compared to the reference due to a phase change at the metal-oxide interface. These oscillations are not detrimental for polarization imaging since the $s$-polarization stays low and the ER is higher than 200 from 650 to $850 \mathrm{~nm}$. The higher ER than for the WGP measured on glass is most probably due to the thicker metal film, which lowers the $s$-polarized transmission (see single slit discussion above). These measurements demonstrate the possibility to integrate efficient WGP for the visible spectrum on CMOS detectors. In contrast to micropolarizers previously fabricated above pixel arrays, these polarizers could be integrated directly within CMOS process due to their planarity and dimensions.

To conclude, efficient WGP have been obtained in the visible spectrum. These polarizers have been integrated on a pixel of a CMOS camera and demonstrate ER of 200. An array of such polarizers with different orientation above the pixel array of a CMOS camera would permit real time polarization imaging.

We would like to thank Dragan Damjanovic from the EPFL for his technical assistance and Edoardo Franzi's group for supplying photodetectors and their support. This work was funded by the European Community under Project No. IST-FP6-034506'PLEAS'.

${ }^{1}$ L. B. Wolff, IEEE Expert 10, 30 (1995).

${ }^{2}$ S. Makita, Y. Yasuno, T. Endo, M. Itoh, and T. Yatagai, Appl. Opt. 45, 1142 (2006).

${ }^{3}$ J. Guo and D. J. Brady, Opt. Eng. (Bellingham) 36, 2268 (1997).

${ }^{4}$ G. P. Nordin, J. T. Meier, P. C. Deguzman, and M. W. Jones, J. Opt. Soc. Am. A Opt. Image Sci. Vis 16, 1168 (1999).

${ }^{5}$ S. Kawakami and Y. Inoue, IEICE Trans. Electron. E90-C, 1046 (2007).

${ }^{6}$ E. Hecht, Optics (Addison-Wesley-Longman, Massachusetts, 1998), p. 319.

${ }^{7}$ H. Hertz, Ann. Phys. Chem. 36, 769 (1889).

${ }^{8}$ G. R. Bird and M. Parrish, Jr., J. Opt. Soc. Am. 50, 886 (1960).

${ }^{9}$ J. P. Auton, Appl. Opt. 6, 1023 (1967).

${ }^{10}$ S. W. Ahn, K. D. Lee, and J. S. Kim, Nanotechnology 16, 1874 (2005).

${ }^{11} \mathrm{H}$. Tamada, T. Doumuki, T. Yamaguchi, and S. Matsumoto, Opt. Lett. 22, 419 (1997).

${ }^{12}$ Y. Takakura, Phys. Rev. Lett. 86, 5601 (2001).

${ }^{13}$ C. Genet and T. W. Ebbesen, Nature (London) 445, 39 (2007).

${ }^{14}$ Y. Pang, C. Genet, and T. W. Ebbesen, Opt. Commun. 280, 10 (2007).

${ }^{15}$ N. D. Arora, L. Song, S. M. Shah, K. Joshi, K. Thumaty, A. Fujimura, L. C. Yeh, and P. Yang, IEEE Trans. Semicond. Manuf. 18, 262 (2005).

${ }^{16}$ H. Lochbihler and R. A. Depine, Appl. Opt. 32, 3459 (1993).

${ }^{17}$ Data may be retrieved at http://www.sopra-sa.com

${ }^{18}$ Q. Cao and P. Lalanne, Phys. Rev. Lett. 88, 057403 (2002).

${ }^{19}$ D. Crouse and P. Keshavareddy, Opt. Express 13, 7760 (2005).

${ }^{20}$ R. W. Wood, Proc. Phys. Soc. London 18, 269 (1902).

${ }^{21}$ P. F. Ruedi, P. Heim, F. Kaess, E. Grenet, F. Heitger, P. Y. Burgi, S. Gyger, and P. Nussbaum, IEEE J. Solid-State Circuits 38, 2325 (2003).

${ }^{22}$ P. Magnan, Nucl. Instrum. Methods Phys. Res. A 504, 199 (2003). 